\title{
Excitation of autoionizing states of helium-like ions by scattering of high-energy particles*
}

\author{
A.I. Mikhailov ${ }^{1}$, A.V. Nefiodov ${ }^{1}$, and G. Plunien ${ }^{2}$ \\ ${ }^{1}$ PNPI, 188300 Gatchina, St. Petersburg, Russia; ${ }^{2}$ TU Dresden, Mommsenstr. 13, D-01062 Dresden, Germany
}

Two-electron excitations of atoms and ions induced by impact of charged particles and photons are fundamental processes, which have been studied since decades. Along with double ionization and ionization accompanied by simultaneous excitation, double excitation is caused by the interelectronic interaction. Accordingly, the cross sections of these processes turn out to be extremely sensitive to the quality of which dynamic correlations are taken into account. This allows one to test different theoretical approaches. In experiments, the neutral helium atom is perferably used as a target, since it is the simplest multielectron system. Helium-like ions with moderate values of nuclear charge $Z$ are even more attractive for investigation of correlated processes, since the corresponding cross sections exhibit a universal scaling behavior. However, such processes are rather rare. Their cross sections decrease rapidly with increasing $Z$ values.

In a most recent paper [1], we have studied two-electron excitations of helium-like ions into the two lower autoionizing $2 s^{2}\left({ }^{1} S\right)$ and $2 p^{2}\left({ }^{1} S\right)$ states (in the following denoted, for simplicity, by numbers 1 and 2 , respectively) by collisions with high-energy electrons and photons. The calculations are performed analytically within the framework of non-relativistic perturbation theory with respect to the interelectronic interaction. The atomic targets are assumed to be characterized by the small parameters $1 / Z \ll 1$ and $\alpha Z \ll 1$, where $\alpha$ is the fine-structure constant. The Auger widths of the levels are equal to $\Gamma_{1}=0.226 \mathrm{eV}$ and $\Gamma_{2}=0.011 \mathrm{eV}$.

In high-energy non-relativistic domain characterized by $3 / 2 \ll \varepsilon \ll 2(\alpha Z)^{-2}$, the total cross section can be cast into the following scaling form $(\hbar=1, c=1)$ [1]:

$$
\sigma^{* *}=\frac{\sigma_{0}}{Z^{6} \varepsilon} B(\varepsilon), \quad B(\varepsilon)=\int_{x_{1}}^{x_{2}} Q(x) d x,
$$

where $\sigma_{0}=\pi a_{0}^{2}=87.974 \mathrm{Mb}$ and $a_{0}=1 /(m \alpha)$ is the Bohr radius. The limits of integration over the dimensionless momentum transfer $x$ are given by $x_{1}=9 /\left(4 x_{2}\right)$ and $x_{2}=(\sqrt{\varepsilon}+\sqrt{\varepsilon-3 / 2})^{2}$, respectively. The dimensionless energy $\varepsilon$ is related to the asymptotic velocity $v$ of projectile according to $\varepsilon=v^{2} /(\alpha Z)^{2}$.

The universal functions $Q(x)$ and $B(\varepsilon)$ are independent of the values of $Z$. The excitation process is characterized by a rather small momentum transfer $x \lesssim 4$ (see Fig. 1). The scalings $B(\varepsilon)$ are saturated already at $\varepsilon \gtrsim 10$. For the lower autoionizing states, the asymptotic high-energy limits amount to the values $B_{1}=0.0231$ and $B_{2}=0.0072$.

\footnotetext{
*Work supported by GSI
}

Then for fast projectiles ( $\varepsilon \gg 3 / 2$ ), the total cross sections have a simple scaling behavior like $\sigma^{* *} \sim \varepsilon^{-1}$, which is similar to that for single-electron excitations $1 s \rightarrow n s$ [2]. Note, that in this case, the formula (1) can also be employed for collisions with heavy charged particles, which are nevertheless much lighter than the atomic nucleus of target. If the charge number of the incident particle is equal to $\pm z$ (in units of the elementary charge $e$ ), then the cross section should be multiplied by the factor $z^{2}$. Although $\Gamma_{2} \ll \Gamma_{1}$, both the autoionizing $2 s^{2}\left({ }^{1} S\right)$ and $2 p^{2}\left({ }^{1} S\right)$ levels can be efficiently excited by high-energy particle scattering.
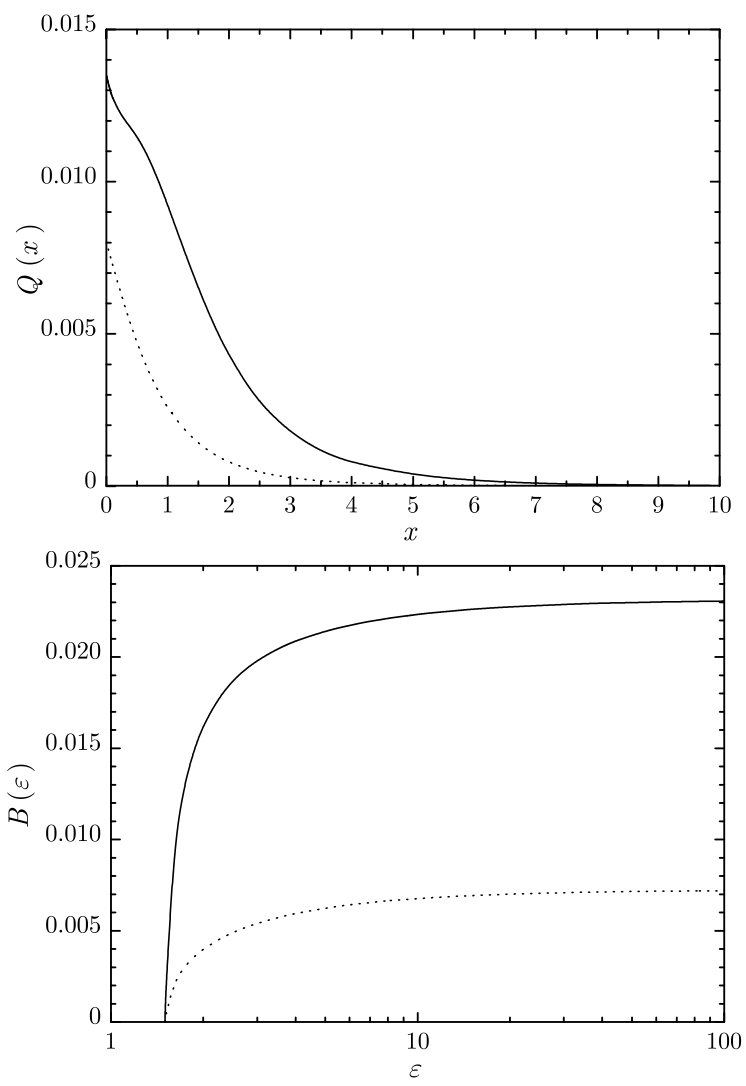

Figure 1: The universal functions $Q(x)$ and $B(\varepsilon)$ : solid line, $2 s^{2}\left({ }^{1} S\right)$ state; dotted line, $2 p^{2}\left({ }^{1} S\right)$ state.

\section{References}

[1] A. I. Mikhailov, A. V. Nefiodov, and G. Plunien, JETP 116 (2013) (in press).

[2] A. V. Nefiodov and G. Plunien, J. Phys. B 43 (2010) 165206. 